\title{
CCL18 promotes the invasion and metastasis of breast cancer through Annexin A2
}

\author{
CHUNLING ZHAO $^{1^{*}}$, SHUXIAN ZHENG $^{2 *}$, ZIQIAN YAN $^{2}$, ZIKUN DENG $^{2}$, \\ RUIGE WANG $^{2}$ and BAOGANG ZHANG ${ }^{2}$
}

Departments of ${ }^{1}$ Cell Biology and ${ }^{2}$ Pathology, Weifang Medical University, Weifang, Shandong 261053, P.R. China

Received November 16, 2018; Accepted August 23, 2019

DOI: 10.3892/or.2019.7426

\begin{abstract}
Chemokine (C-C motif) ligand 18 (CCL18) is derived from breast tumor-associated macrophages (TAMs), which are primarily a macrophage subpopulation with an M2 phenotype. CCL18 binds to its receptor, PYK2 N-terminal domain interacting receptor 1 (Nirl), and promotes tumor progression and metastasis by inducing epithelial-mesenchymal transition (EMT) via the PI3K/Akt/GSK3 $\beta /$ Snail signaling pathway in breast cancer cells. Recent research shows that Annexin A2 (AnxA2) plays a significant role in the invasion, metastasis, angiogenesis, proliferation, F-actin polymerization and multidrug resistance to chemotherapy of breast cancer. The present study aimed to elucidate the molecular mechanisms by which CCL18 promotes breast cancer progression through AnxA2 which are not fully understood. Western blot analysis showed that the expression of AnxA2 was upregulated in highly invasive breast cancer cell lines and invasive ductal carcinoma. Furthermore, through chemotaxis, scratch, Matrigel invasion, and spontaneous metastasis assays, it was demonstrated that Anx A2 enhanced the invasion of breast cancer cells and the metastasis of human breast cancer cells to lungs of SCID mice with CCL18 stimulation. Cellular F-actin measurement assay showed that reduction of AnxA2 suppressed CCL18-induced F-actin polymerization though phosphorylation of integrin $\beta 1$ in breast cancer cells. Immunofluorescence and western blot analysis revealed that AnxA2 promoted CCL18-induced EMT via the PI3K/Akt/GSK3 $\beta /$ Snail signaling pathway, and LY294002 inhibited the phosphorylation of AnxA2 in vitro. In brief, AnxA2, as a downstream molecule of Nir 1 binding to CCL18, promotes invasion and metastasis by EMT through the PI3K/Akt/GSK3 $\beta /$ Snail signaling pathway in breast cancer. This study suggests that AnxA2 is a potential
\end{abstract}

Correspondence to: Professor Baogang Zhang, Department of Pathology, Weifang Medical University, 7166 Baotong Street, Weifang, Shandong 261053, P.R. China

E-mail: zhangbg@wfmc.edu.cn

*Contributed equally

Key words: annexin A2, CCL18, invasion, metastasis, breast cancer anti-invasion/metastasis target for therapeutic intervention in breast cancer.

\section{Introduction}

Breast cancer is the most commonly diagnosed malignant tumor in women worldwide. Despite the numerous and advanced types of combined therapy, breast cancer remains among the main causes of tumor-related deaths in women (1). Breast cancer is associated with aggressive local invasion, metastasis in advanced stage and multidrug resistance to chemotherapy (2). Various molecular markers have been identified that contribute to the regulation of breast cancer invasion and metastasis, such as cell cycle regulators and cell-adhesion proteins (3).

The binding of chemokines to specific G-protein-coupled receptors induce cytoskeletal rearrangement and directional migration and then critically regulate tumor cell migration and metastasis (4). Chemokines were initially characterized due to their association with inflammatory responses. These are members of a superfamily of chemotactic cytokines (5). Chemokine (C-C motif) ligand 18 (CCL18) is a type of chemokine, which is predominantly derived from breast tumor-associated macrophages (TAMs) (6). Previous data indicate that the binding of CCL18 to its receptor, PYK2 $\mathrm{N}$-terminal domain interacting receptor 1 (Nirl), promotes the invasion, metastasis and EMT of breast cancer cells through the PI3K/Akt/GSK3 $\beta /$ Snail signaling pathway $(3,7)$. However, the involvement of other factors in the CCL18-induced migration, invasion and metastasis of breast cancer cells remains unclear.

AnnexinA2 (AnxA2), a member of the calcium-dependent phospholipid binding proteins, plays a crucial role in cancer progression $(8,9)$. AnxA2 is reported to be overexpressed in a variety of cancers and contributes to invasion (10-12), metastasis $(11,13)$, angiogenesis $(14)$, proliferation $(13,15-17)$, F-actin polymerization (16), multidrug resistance to chemotherapy (18-20) and epithelial-mesenchymal transition (EMT) (9).

EMT is crucial in embryonic development and cancer progression and it is a process during which epithelial-like cancer cells transform into mesenchymal-like tumor cells with upregulation of mesenchymal markers and downregulation of epithelial markers. Moreover, EMT is a vital contributor 
to the invasion and metastasis of breast cancer cells $(21,22)$. In the present study, we detected the association of AnxA2 expression in breast tissue specimens with the clinicopathological characteristics of patients and observed the role of AnxA2 expression in breast cancer migration and invasion under the induction of rCCL18 to verify the hypothesis that AnxA2 promotes CCL18-induced progression and metastasis of breast cancer cells. In our reseach, AnxA2 was found to contribute to CCL18-induced phosphorylation of integrin $\beta 1$ in breast cancer cells, which is a crucial step in cytoskeletal rearrangement and cell adhesion.

We hypothesized that the binding of CCL18 to Nir1 promotes tumor progression and metastasis by inducing EMT via the PI3K/Akt/GSK3 $\beta /$ Snail signaling pathway though AnxA2 in vitro and in vivo.

\section{Materials and methods}

Patients and tissue specimens. All tissue specimens were obtained from the Department of Pathology, Affiliated Hospital of Weifang Medical University from 1 January 2012 to 31 December 2016. These tissue specimens consisted of samples from 120 cases of invasive ductal carcinoma and their corresponding adjacent non-tumor tissues. The distance between invasive ductal carcinoma and their corresponding adjacent non-tumor tissues was $>5 \mathrm{~cm}$. The mean age of the patients was 47.3 years (range, 35-76). All tissue specimens were fixed, embedded in paraffin, serial sectioned and H\&E stained and finally stored at room temperature. The inclusion criteria for the patients was a diagnosis of breast cancer and agreement to participate in the present study. Patient samples were collected according to a protocol approved by the Institutional Review Board, and patients provided signed consent for use of their tissue specimens in the present study. The study protocol was reviewed and approved by the Weifang Medical University Ethics Committee (approval no. 045, 26-Feb-2016). Clinical information of the samples is documented in detail in Table I.

Cell lines and reagents. The human breast cancer cell lines, MDA-MB-231, T47D and MCF-7, were obtained from the American Type Culture Collection (ATCC). Lipofectamine 2000 was obtained from Invitrogen; Thermo Fisher Scientific, Inc. Plasmid construction was carried out by Genescript (USA). The Pierce ${ }^{\mathrm{TM}}$ BCA Protein Assay Kit and the Pierce ${ }^{\mathrm{TM}}$ ECL kit were purchased from Thermo Fisher Scientific, Inc. Primary antibodies including anti-AnxA2 (dilution 1:1,000; cat. no. ab178677; Abcam), anti-phospho-AnxA2 (1:1,000; cat. no. sc-135753; Santa Cruz Biotechnology), anti-Akt (dilution 1:1,000; cat. no. 2920; Cell Signaling Technology), anti-phospho-Akt-Ser473 (dilution 1:1,000; cat. no. 15116; Cell Signaling Technology), anti-phospho-Akt-Thr308 (dilution 1:1,000; cat. no. 13038; Cell Signaling Technology), anti-integrin $\beta 1$ (dilution 1:1,000; cat. no. 34971; Cell Signaling Technology), anti-phospho-integrin $\beta 1$ (dilution 1:1,000; cat. no. 44-873G; Thermo Fisher Scientific, Inc.), anti-GSK3 $\beta$ (dilution 1:1,000; cat. no. 12456; Cell Signaling Technology), anti-phospho-GSK3 $\beta$ (dilution 1:1,000; cat. no. 9323; Cell Signaling Technology), anti-E-cadherin (dilution 1:1,000; cat. no. 7687; Cell Signaling Technology), anti-N-cadherin (dilution 1:1,000; cat. no. 13116; Cell Signaling Technology), anti-vimentin (dilution 1:1,000; cat. no. 3390; Cell Signaling Technology), anti-Snail (1:1,000; cat. no. 3895; Cell Signaling Technology), $\beta$-actin (dilution 1:1,000; cat. no. 3700; Santa Cruz Biotechnology), anti-Nucleolin (dilution 1:1,000; cat. no. ab22758; Abcam) and the anti-mouse, rabbit and goat secondary antibodies were obtained from Santa Cruz Biotechnology, Inc. Fluorochrome-conjugated secondary antibodies, Alexa Flour 594 and TRITC, (Invitrogen; Thermo Fisher Scientific, Inc.) were diluted 1:600 in blocking buffer (Solarbio). All culture media and related reagents were purchased from Hyclone/GE Healthcare, USA.

Animals. Forty 1-week-old female SCID mice were purchased from Wei Tong Li Hua Experimental Animal Co. (initial mean weight $18.3 \mathrm{~g}$; range $16.3-19.7 \mathrm{~g}$ ). The mice were maintained in laminar flow rooms under constant temperature and humidity. The food and water of mice were sterilized, and the food and water were renewed ad libitum.

Plasmid construction, shRNA and plasmid transfection. Cells were cultured in a 6 -well plate for $24 \mathrm{~h}$ in complete medium before transfection. Transfection was performed with Lipofectamine 2000 according to the manufacturer's instructions. Sequences of AnxA2 siRNA were 5'-GGTCTG AATTCAAGAGAAA-3' and 5'-GCCAAAGAAATGAAC ATTC-3'. Sequences of Nir 1 siRNA were 5'-GGGAGA AGUGGCUUCGUAATT-3' and 5'-UUACGAAGCCAC UUCUCCCGG-3'. Sequences of Akt siRNA were 5'-GCU GGCUCCACAAACGUGGUGAAUA-3' and 5'-UAUUCA CCACGUUUGUGGAGCCAGC-3'. Culture medium with $600 \mathrm{ng} / \mathrm{ml}$ hygromycin B was used to select stably transfected cells. The MDA-MB-231 cells were stably transfected with the sequences of AnxA2 siRNA, Nir 1 siRNA or Akt siRNA respectively marked as SiAnxA2/MDA-231, SiNir1/MDA-231 and SiAkt/MDA-231. A scrambled SiRNA was used as a control (Scr/MDA-231). Surviving cells were evaluated for AnxA2 expression by western blot analysis. For plasmid transfection, cells were plated in a 6 -well plate for $24 \mathrm{~h}$ before transfection. AnxA2 cDNA was cloned in the KpnI-XbaI sites of pcDNA3.1, and was confirmed by DNA sequencing. MCF-7 cells were transfected with the pcDNA3.1-AnxA2 plasmid, pcDNA3.1-Nir1 plasmid, pcDNA3.1-Akt plasmid or pcDNA3.1 vector using Lipofectamine 2000 and marked as AnxA2/MCF-7, NIR1/MCF-7, Akt/MCF-7 or Con/MCF-7. Stable transfected cells were selected and maintained in culture medium with G418.

Western blot analysis. Total cellular proteins were extracted with ice-cold RIPA cell lysis buffer with Protease Inhibitor Cocktail. We employed the BCA Protein Assay Kit (Pierce) to quantify the protein concentration. The amount of protein loaded in each lane is $10 \mu \mathrm{l}$. Lysates were separated by $10 \%$ SDS-PAGE and transferred onto a PVDF membrane. The membrane was incubated in primary antibodies, horseradish HRP-conjugated anti-rabbit IgG secondary antibody successively. Western blots were visualized using an ECL kit (Pierce; Thermo Fisher Scientific, Inc.) according to the manufacturer's protocol. $\beta$-actin was served as an internal loading control for each blot. The intensity of the detected bands was analyzed using an ImageJ software (version 1.42; National Institutes of Health). 
Table I. Association between AnxA2 expression and clinical features of the invasive ductal carcinoma patients enrolled in this study.

\begin{tabular}{|c|c|c|c|}
\hline \multirow[b]{2}{*}{ Variables } & \multicolumn{2}{|c|}{$\begin{array}{c}\text { AnxA2 } \\
\text { expression }\end{array}$} & \multirow[b]{2}{*}{ P-value } \\
\hline & High & Low & \\
\hline \multicolumn{4}{|c|}{ Age (years) } \\
\hline$\leq 50$ & 34 & 19 & 0.190 \\
\hline$\geq 51$ & 35 & 32 & \\
\hline \multicolumn{4}{|l|}{ Tumor size } \\
\hline$\leq 5 \mathrm{~cm}$ & 31 & 23 & 0.723 \\
\hline$>5 \mathrm{~cm}$ & 40 & 26 & \\
\hline \multicolumn{4}{|c|}{ Tumor differentiation } \\
\hline I & 17 & 21 & 0.036 \\
\hline II & 25 & 16 & \\
\hline III & 30 & 11 & \\
\hline \multicolumn{4}{|c|}{ Lymph node metastasis } \\
\hline Yes & 48 & 21 & 0.007 \\
\hline No & 23 & 28 & \\
\hline \multicolumn{4}{|c|}{ Distant metastasis } \\
\hline Yes & 46 & 20 & 0.009 \\
\hline No & 25 & 29 & \\
\hline \multicolumn{4}{|l|}{ Nir1 } \\
\hline Positive & 55 & 24 & 0.006 \\
\hline Negative & 18 & 23 & \\
\hline
\end{tabular}

AnxA2, annexin A2. P-values in bold print indicate statistically significant results.

Wound healing/scratch assay. Wound healing/scratch assays were carried out as previously described (23). In brief, cells were plated in 6-well plates and cultured to a monolayer. Next, a scratch was created in the middle using a $10-\mu 1$ pipette tip. The medium was replaced by $1 \%$ bovine serum albumin (BSA) in RPMI medium. The cells were incubated at $37^{\circ} \mathrm{C}$ in a sterile incubator, and the wound trace was photographed at $0,5,10,15$ and $20 \mathrm{~h}$. The distance of the scratch (in $\mathrm{mm}$ ) was measured under a light microscope at magnification x200. All samples were examined in triplicate, and the data are shown as the means.

Invasion assay. To evaluate the cell invasion ability, Transwell assays were carried out as previously described $(3,23)$. In brief, the cell suspension at a final concentration of $4 \times 10^{5} / \mathrm{ml}$ was added into the upper Transwell inserts precoated with Matrigel, and then the lower insert was loaded with $10 \%$ FBS in cell culture medium. After $24 \mathrm{~h}$ of incubation at $37^{\circ} \mathrm{C}$ in a sterile incubator, the invaded cells were fixed. The invaded cells were stained with $0.2 \%$ crystal violet at room temperature for $10 \mathrm{~min}$. The number of invaded cells was counted under an inverted microscope. All samples were examined in triplicate, and the data are showed as the means.
Chemotaxis assay. Chemotaxis assay was performed as described previously $(3,23)$. Briefly, a polycarbonate filter membrane was inserted between the upper and lower chambers. After pretreatment with $10 \mu \mathrm{g} / \mathrm{ml}$ fibronectin overnight, it was dried in air. CCL18, as chemoattractant, was added into the lower chamber and the cell suspension at a final concentration of $5 \times 10^{5} / \mathrm{ml}$ was added to the upper chambers. Then, the chamber was incubated at $37^{\circ} \mathrm{C}$ in a sterile incubator for $3 \mathrm{~h}$. The filter membrane was washed, fixed and stained. The number of migrating cells was calculated using a microscope at a magnification $\mathrm{x} 400$ in three random fields. Chemotaxis index $=$ the number of migrating cells in the chemoattractant gradient/the number of migrating cells in the medium control. All samples were tested in triplicate and the data are shown as the means.

Adhesion assay. The adhesion assays was conducted as described previously (23). The cells suspension at a final concentration of $3 \times 10^{5} / \mathrm{ml}$ incubated at $37^{\circ} \mathrm{C}$ in a sterile incubator for $20 \mathrm{~min}$, and then, cells were promptly added to a 24-well plate containing dried glass coverslips with or without $10 \mathrm{ng} / \mathrm{ml} \mathrm{rCCL} 18$. The coverslips were pretreated with fibronectin and then dried for $30 \mathrm{~min}$. After 5, 15 and $30 \mathrm{~min}$ of incubation, the cells were washed and fixed. The number of cells attached to the coverslips were calculated using a light microscope at magnification of $\mathrm{x} 400$.

Cellular F-actin measurement. F-actin polymerization assay was performed as previously described $(4,24)$. In brief, cells were cultured for $24 \mathrm{~h}$ in complete medium and cultured in binding medium for $2 \mathrm{~h}$. Cells were fixed, permeabilized, and stained in the dark with TRITC-labelled Phalloidin, which was

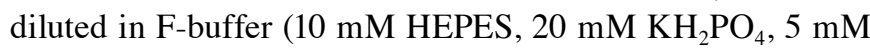
EGTA, $2 \mathrm{mM} \mathrm{MgCl}_{2}$, Dulbecco's PBS, pH 6.8) for $30 \mathrm{~min}$. Cells was measured by fluorescence analysis. The F-actin content was calculated by the following equation: F-actin $\Delta \mathrm{t} / \mathrm{F}$-actin $0=($ fluorescence $\Delta \mathrm{t} / \mathrm{mg}$ per $\mathrm{ml} /$ fluorescence $0 / \mathrm{mg}$ per $\mathrm{ml}$ ). All experiments were repeated at least three times.

Immunofluorescence. Immunofluorescence was performed as previously described (3). Briefly, the cells were cultured in 24-well plates containing sterile coverslips $24 \mathrm{~h}$ before this experiment, and then starved overnight. After stimulation with $10 \mathrm{ng} / \mathrm{ml} \mathrm{rCCL18,} \mathrm{cells} \mathrm{were} \mathrm{washed,} \mathrm{fixed} \mathrm{and} \mathrm{permeabilized}$ as previously described (3). Cells were incubated in primary antibody overnight at $4^{\circ} \mathrm{C}$. Negative control comprised rabbit IgG. The cells were then directly incubated at room temperature for $2 \mathrm{~h}$ in dark with Alexa Fluor 594-conjugated secondary antibodies and 4',6-diamidino-2-phenylindole (DAPI) in the dark. The results were analyzed using fluorescence microscopy. All experiments were repeated at least three times.

In vivo spontaneous metastasis assay. Metastasis assays were performed as described previously (3). Forty SCID mice were randomly divided into four groups. In total, the Scr/MDA-231

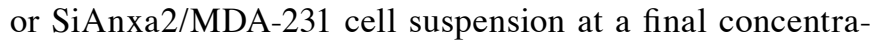
tion of $2 \times 10^{6} / \mathrm{ml}$ was injected into the mammary fat pads of the mice. When the xenografts were $0.5 \mathrm{~cm}$ in diameter, an intratumoral injection of $100 \mathrm{ng} / \mathrm{kg} \mathrm{rCCL} 18$ or the same volume of vehicle was administered biweekly for 4 continuous 
weeks. After 8 weeks, the mice were sacrificed by cervical dislocation, and the lung surface nodules were counted. The lung tissues were fixed, embedded in paraffin, serial sectioned and H\&E stained to detect lung micrometastasis. The study protocol for the animal study was reviewed and approved by Weifang Medical University Ethics Committee (approval no. 045, 26-Feb-2016).

Statistical analyses. Data are presented as mean \pm SEM from a representative experiment; each assay was independently repeated at least three times. All statistical analyses were performed using SPSS v17.0 software (SPSS, Inc.). Student's t-test or one-way analysis of variance (ANOVA) (SigmaPlot; SigmaStat; Jandel Scientific Software) was used for comparisons between groups, and the relationship between the clinicopathologic characteristics and AnxA2 expression was determined using Chi-square test, with $\mathrm{P}<0.05$ considered statistically significant.

\section{Results}

Expression of AnxA2 in breast cancer and the correlation between AnxA2 and clinicopathological characteristics of the breast cancer cases. Comparative evaluation was carried out of the AnxA2 expression of the paired invasive ductal carcinoma tissues and adjacent non-tumor tissues by western blot analysis. The coupled tissues were obtained from the same patient. Our results showed that the protein level of AnxA2 was overexpressed in invasive ductal carcinoma tissues when compared with that in the coupled adjacent non-tumor tissues (Fig. 1A, left image). To further investigate the correlation between AnxA2 and the clinicopathological characteristics of the breast cancer cases, we assessed the expression of AnxA2 in 120 archived paraffin-embedded specimens of invasive ductal carcinoma and the coupled adjacent non-tumor tissues by qRT-PCR. Our experimental results indicated that the expression of AnxA2 in the invasive ductal carcinoma tissues was significantly associated with tumor differentiation, lymphatic metastasis, distant metastasis and Nir1 expression, but it was independent of age and tumor size (Table I).

As our results showed that the protein level of AnxA2 was overexpressed in invasive ductal carcinoma tissues compared with that in the coupled adjacent non-tumor tissues, we assessed the AnxA2 level in breast cancer cell lines. The protein level of AnxA2 was diverse in the breast cancer cell lines. Highly invasive cells (MDA-MB-231) expressed a higher level of AnxA2 than the low-invasive cells (T47D and MCF-7) (Fig. 1A, right image). Furthermore, CCL18 affected the phosphorylation levels of AnxA2 in breast cancer cells in a time-dependent manner, but not the expression of AnxA2 in MCF-7 and MDA-MB-231 cells (Fig. 1B).

AnxA2 contributes to CCL18-induced chemotaxis in breast cancer cells. We used siRNA to inhibit Nirl or AnxA2 expression to determine the specific function of AnxA2 breast cancer cells. Tygromycin B was used for selecting the successfully transfected cells that stably downregulated the expression of Nir1 or AnxA2. SiRNAs (SiAnxA2\#1 and SiAnxA2\#2) were designed to target Nir1 or AnxA2. Scr/MDA-231 cells were used as control cells that were transfected with a scrambled sequence. We chose SiNir1/MDA-231 and SiAnxA2/MDA-231 cells as the representatives.

Simultaneously, AnxA2 or Nir1 stably transfected cell clones were generated by pcDNA3.1-AnxA2 or pcDNA3.1-Nir1 plasmid subsequent selection. Stable transfected cells were obtained by using medium (culture medium with $600 \mathrm{ml} \mathrm{G} 418$ ). Hence, we decided to present the results from clone 4, designated as AnxA2/MCF-7 and Nir1/MCF-7 cells. Con/MCF-7 cells were used as vector control cells that were transfected with a pcDNA3.1 vector. We performed western blot analysis to identify the protein and phosphorylation levels of AnxA2 in Scr/MDA-231, SiNir1/MDA-231, Con/MCF-7, and Nir1/MCF-7 cells with or without rCCL18 stimulation. Our results indicated that the expression of Nir1 affected CCL18-induced AnxA2 phosphorylation. In other words, CCL18 binding to Nir1 promoted the phosphorylation levels of AnxA2 in breast cancer cells, but not the expression of AnxA2 (Fig. 1C). We performed western blot analysis to identify AnxA2 in stably transfected cell clones. The results are illustrated in Fig. 1D and F. We performed chemotaxis assay to determine whether AnxA2 contributed to CCL18-induced breast cancer cell chemotaxis. The CCL18-induced chemotaxis ability of the diverse breast cancer cell lines followed a representative bell-shaped response curve (Fig. 1E and G). These chemotaxis assay results indicated that AnxA2 promotes CCL18-induced chemotaxis in breast cancer cells.

Decrease in AnxA2 suppresses adhesion, migration and invasion in breast cancer cells. Migration, invasion and proliferation are correlated with a highly aggressive phenotype in breast cancer. Previous studies $(9,17,18,25,26)$ suggest that AnxA2 promotes invasion, migration, proliferation and adhesion in cancer cells. Moreover, chemokine-induced adhesion contributes to cell movement (26). We aimed to determine the influence of AnxA2 on the adhesion in breast cancer cells with or without CCL18 stimulation. Our results revealed that treatment with $10 \mathrm{ng} / \mathrm{ml}$ of rCCL18 promoted the adhesion, which was significantly impaired in the SiAnxA2/MDA-231 cells (Fig. 2A). The results indicated that AnxA2 promoted CCL18-induced cell adhesion. To identify whether AnxA2 affects CCL18-induced migration and invasion in breast cancer cells, we carried out scratch assays and Transwell invasion assays. When a wound was created in the cells, the distance of the SiAnxA2/MDA-231 cell migration was more narrow than the Scr/MDA-231 cells with rCCL18 stimulation (Fig. 2B). The number of SiAnxA2/MDA-231 cells that invaded the Matrigel were considerably fewer than the Scr/MDA-231 cells with $10 \mathrm{ng} / \mathrm{ml} \mathrm{rCCL} 18$ stimulation after $24 \mathrm{~h}$, and there was no obvious difference in the invasion between SiAnxA2/MDA-231 and Scr/MDA-231 cells without rCCL18 stimulation at $24 \mathrm{~h}$ (Fig. 2C). These results showed that suppression of AnxA2 inhibited CCL18-induced adhesion, migration and invasion in breast cancer cells.

Reduction in AnxA2 suppresses CCL18-induced F-actin polymerization in breast cancer cells. The migration and chemotaxis of cells depend on the actin-based cytoskeleton $(27,28)$. The process of F-actin polymerization is quick and transient (29). To testify the hypothesis that the downregulation of AnxA2 could suppress CCL18-induced chemotaxis 
A

Patient
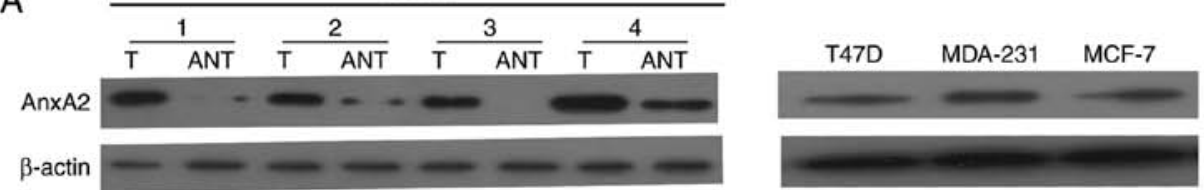

B

MCF-7
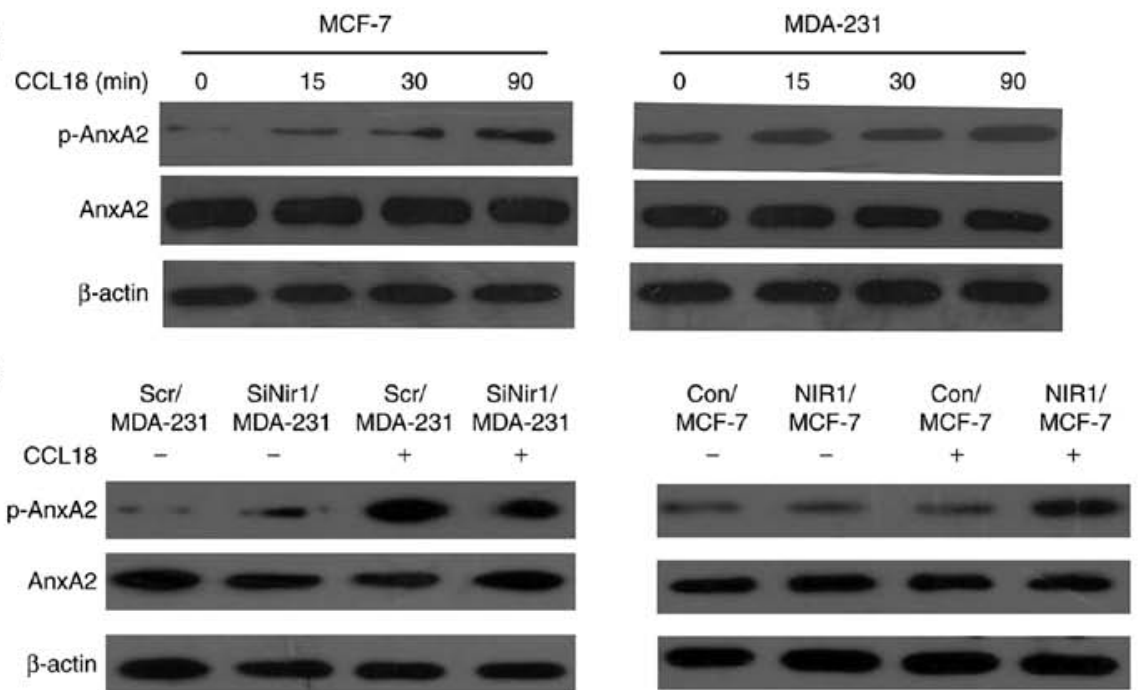

D
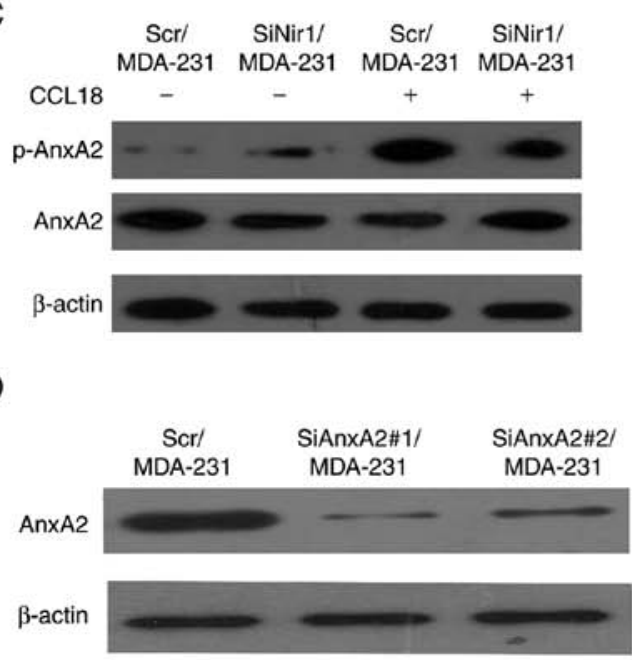

E

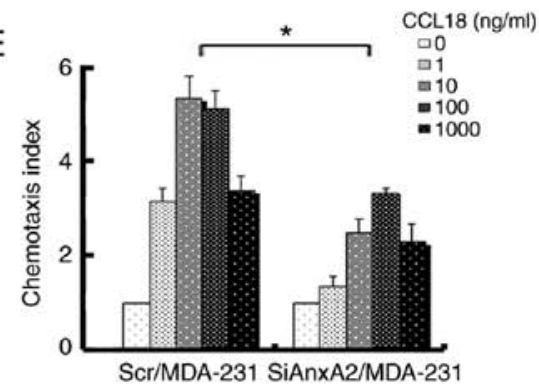

$\mathrm{F}$

G
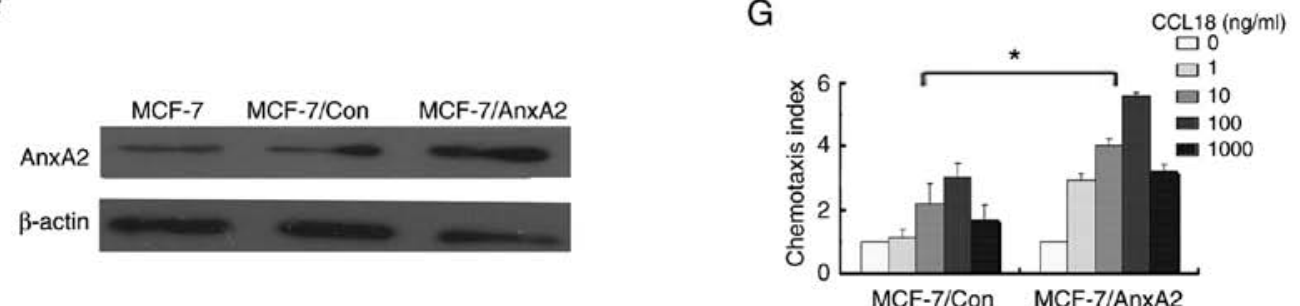

Figure 1. AnxA2 expression in samples from breast cancer patients and breast cancer cell lines. (A) Left panels: Expression of AnxA2 protein in coupled breast cancer tissues (T) and adjacent tissues (ANT), with each pair obtained from the same patient. Right panels: Expression of AnxA2 protein in breast cancer cell lines (T47D, MDA-MB-231, MCF-7). $\beta$-actin was used as a loading control. The results are representative of at least three indenpent experiments. (B) Expression levels of AnxA2 protein in MDA-MB-231 and MCF-7 cell lines upon $10 \mathrm{ng} / \mathrm{ml} \mathrm{rCCL} 18$ stimulation for 0, 15, 30 and $90 \mathrm{~min}$. $\beta$-actin was used as a loading control. (C) Left panels: Expression levels of AnxA2 protein in Scr/MDA-231 and SiNir1/MDA-231 cells with or without $10 \mathrm{ng} / \mathrm{ml} \mathrm{rCCL} 18$ stimulation were detected by western blot analysis. Right panels: Expression levels of AnxA2 protein in Con/MCF-7 and Nirl/MCF-7 cells with or without $10 \mathrm{ng} / \mathrm{ml} \mathrm{rCCL18} \mathrm{stimulation} \mathrm{were} \mathrm{detected} \mathrm{by} \mathrm{western} \mathrm{blot} \mathrm{analysis.} \beta$-actin was used as a loading control. (D) Expression of AnxA2 protein in MDA-MB-231 cells transfected with a scrambled SiRNA as a control (Scr/MDA-231) and with two sets of stable SiRNA-targeting AnxA2 (SiAnxA2\#1/MDA-231 and SiAnxA2\#2/MDA-231) was detected by western blot analysis. $\beta$-actin was used as a loading control. (E) Comparison of chemotactic responses with rCCL18 stimulation in Scr/MDA-231 and SiAnxA2/MDA-231 (SiAnxA2\#1/MDA-231) cells. Columns, mean of triplicate measurements; Bars, standard deviation. ${ }^{*} \mathrm{P}<0.05$ (two-way ANOVA). (F) Expression of AnxA2 protein in Con/MCF-7 and AnxA2/MCF-7 (stable clone 2) cells was detected by western blot analysis. $\beta$-actin was used as a loading control. (G) Comparison of chemotactic responses with rCCL18 stimulation in Con/MCF-7 and AnxA2/MCF-7 cells. The data were collected from a representative of at least three independent experiments. Columns, mean of triplicate measurements; bars, standard deviation. ${ }^{*} \mathrm{P}<0.05$ (two-way ANOVA). CCL18, chemokine (C-C motif) ligand 18; Nir1, PYK2 N-terminal domain interacting receptor 1; AnxA2, Annexin A2.

of breast cancer cells by suppressing F-actin polymerization, we performed F-actin polymerization assay. The results indicated that CCL18 elicited transient actin polymerization at 15 and $60 \mathrm{~min}$ in the Scr/MDA-231 cells. Actin polymerization was significantly decreased in the SiAnxA2/MDA-231 cells (Fig. 3A), which suggested that AnxA2 plays a vital role in regulating cytoskeleton rearrangement with CCL18 stimulation. Immunofluorescent staining was used to detect the F-actin content. rCCL18 increased the F-actin content in the Scr/MDA-231 cells, but not in SiAnxA2/MDA-231 cells 
A
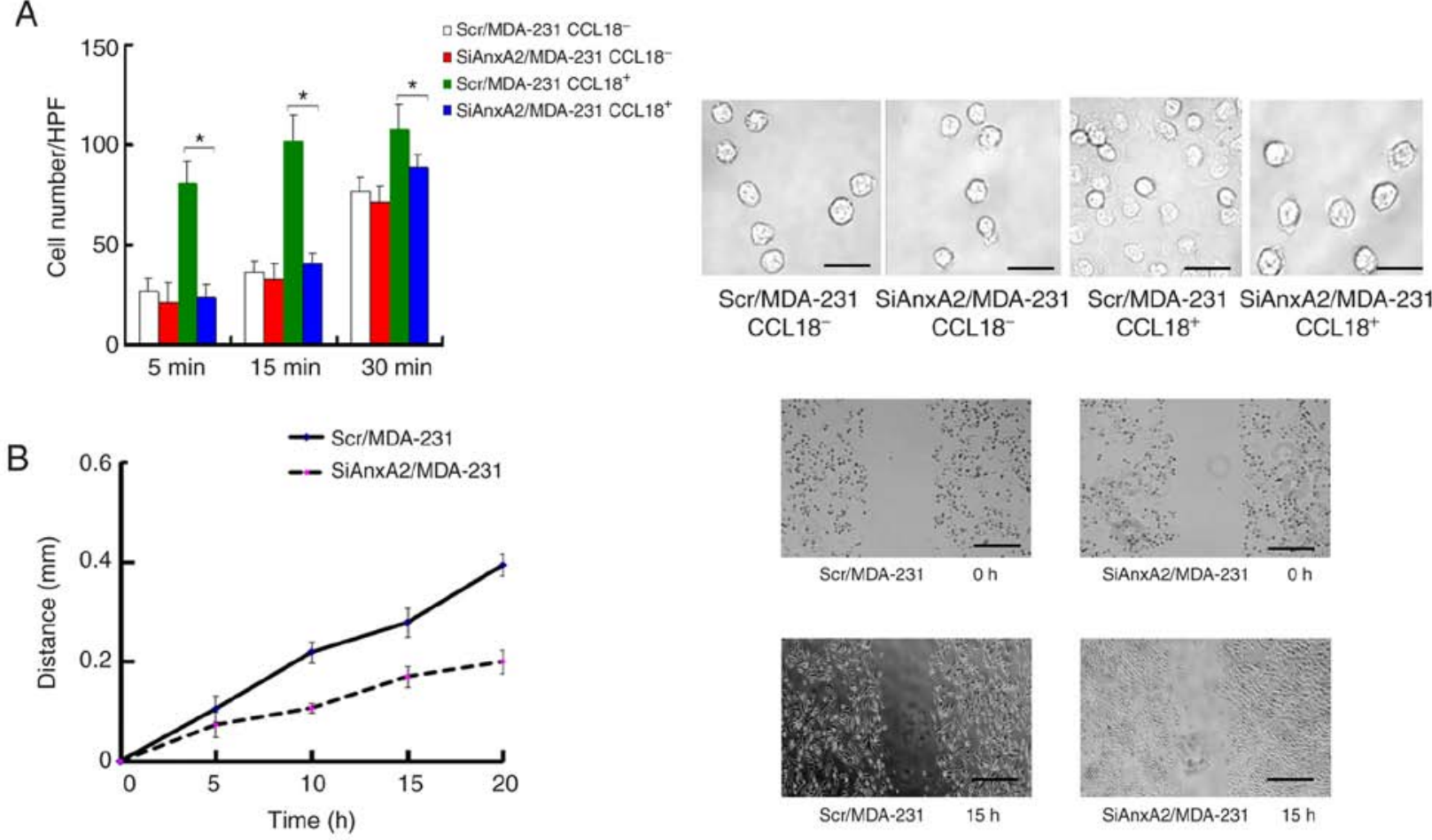

C
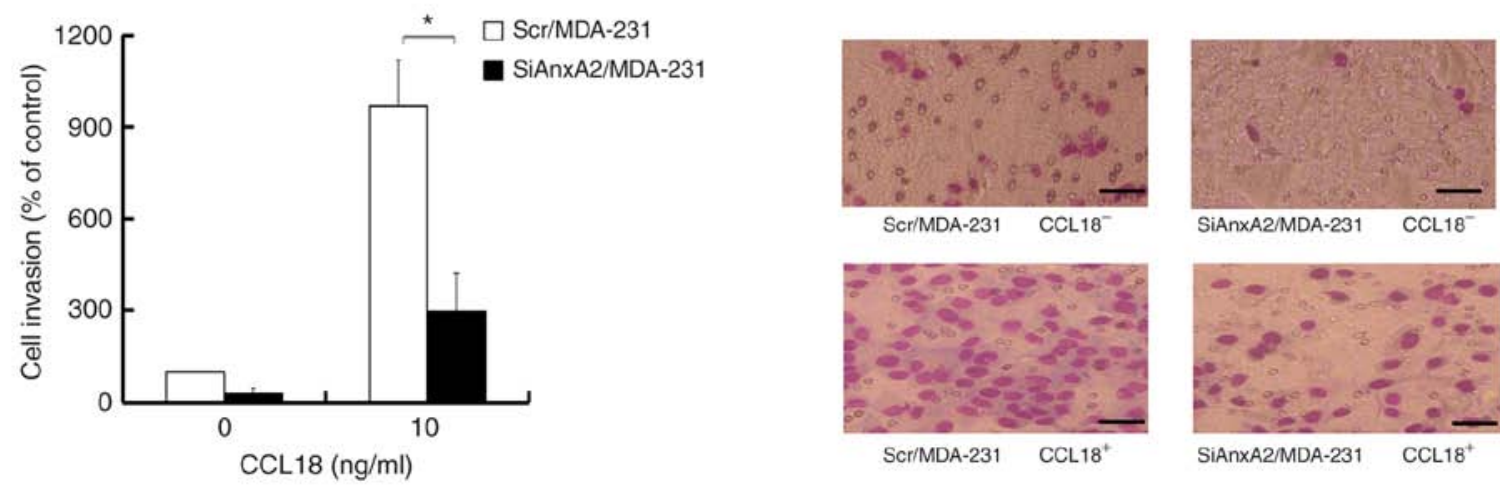

Figure 2. AnxA2 promotes CCL18-induced chemotaxis migration and invasion of breast cancer cells. (A) Left histogram: Comparison of cell adhesion in Scr/MDA-231 and SiAnxA2/MDA-231 (SiAnxA2\#1/MDA-231) cells with $\left(C C L 18^{+}\right)$or without (CCL18) $10 \mathrm{ng} / \mathrm{ml}$ rCCL18 stimulation. Columns, mean of triplicate measurements; Bars, standard deviation. ${ }^{*} \mathrm{P}<0.05$ (two-way ANOVA). Right panels: Images were taken at a x 400 magnification. Scale bar, $20 \mu \mathrm{m}$. (B) Left graph: Quantification of the in vitro scratch assays in Scr/MDA-231 and SiAnxA2/MDA-231 cells. The distance of cell migration was measured. Standard deviation is shown on the graph. Right: Images were photographed at 0 and $20 \mathrm{~h}$. Scale bars, $400 \mu \mathrm{m}$. (C) The CCL18-induced invasion in Scr/MDA-231 and SiAnxA2/MDA-231 cells was analyzed with or without rCCL18 stimulation. Left histogram: Quantification of invaded cells were analyzed. Columns, mean of triplicate measurements; bars, standard deviation. " $\mathrm{P}<0.05$ (two-way ANOVA). Right: Images were captured at a x400 magnification. Scale bar, $20 \mu \mathrm{m}$.

(Fig. 3B). Integrin $\beta 1$ binding to fibronectin plays a crucial part in adhesion of breast cancer cells (30). Thus, western blot analysis was performed to detect the CCL18-induced activation of integrin $\beta 1$. As shown in Fig. 3C, the CCL18-induced phosphorylation of integrin $\beta 1$ was distinctly impaired in the SiAnxA2/MDA-231 cells, in accordance with a reduction in adhesion. The results indicated that AnxA2 regulated migration and invasion directly by modulating the CCL18-induced adhesion and F-actin polymerization in the MDA-MB-231 cells.

AnxA2 promotes CCL18-induced EMT via the Akt/GSK3 $\beta$ signaling pathway. EMT is a well-coordinated process during cancer progression, which is related to the metastatic potential of tumor cells. Our previous study (3) indicated that CCL18 binding to Nirl enhances the invasion ability of breast cancer cells through the PI3K/Akt/GSK3 $\beta /$ Snail signaling pathway. We assumed that AnxA2, as the downstream molecule of CCL18 that binds to Nir1, promotes progression and metastasis by EMT of breast cancer cells through the PI3K/Akt/GSK3 $\beta /$ Snail signaling pathway in breast cancer cells. To investigate the role of AnxA2 in the promotion of migration and metastasis in breast cancer cells through EMT, we performed western blot assays and immunofluorescence to detect vimentin, N-cadherin (mesenchymal marker), and E-cadherin (epithelial marker) in cells with or without $10 \mathrm{ng} / \mathrm{ml} \mathrm{rCCL18} \mathrm{stimulation} \mathrm{for} 3$ days. Western blot assays showed that compared with the Scr/MDA-231 cells, the expression levels of $\mathrm{N}$-cadherin and vimentin were distinctly lower and the expression of E-cadherin was higher in the SiAnxA2/MDA-231 cells with $10 \mathrm{ng} / \mathrm{ml} \mathrm{rCCL18} \mathrm{for}$ 3 days (Fig. 4A, left panels). In AnxA2/MCF-7 cells, the 

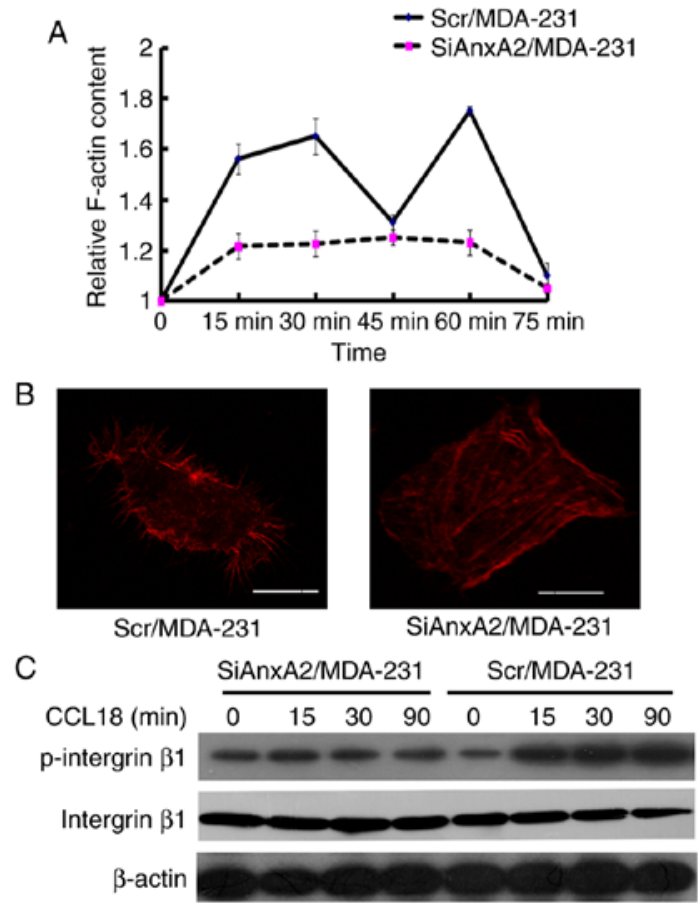

Figure 3. AnxA2 enhances CCL 18-induced actin polymerization in breast cancer cells. (A) Time course of relative F-actin content in Scr/MDA-231 and SiAnxA2/MDA-231 cells with CCL18 stimulation. CCL18, $10 \mathrm{ng} / \mathrm{ml}$. The data were collected from a representative of at least three repeated experiments. Each data point was an average of triplicate assays. Bars, standard deviation. (B) Cytoskeleton rearrangement in Scr/MDA-231 and SiAnxA2/MDA-231 cells was imaged by confocal microscopy. Cells were treated with $10 \mathrm{ng} / \mathrm{ml}$ rCCL18. Figures show representative images from three repeated experiments. Scale bars, $5 \mu \mathrm{m}$. (C) Western blot analysis of phosphorylated (p-)integrin $\beta 1$ and integrin $\beta 1$, in total cell lysates from Scr/MDA-231 and SiAnxA2/MDA-231 cells upon $10 \mathrm{ng} / \mathrm{ml} \mathrm{rCCL18} \mathrm{stimula-}$ tion for $0,15,30$ and $90 \mathrm{~min}$. $\beta$-actin was used as a loading control. The results of the western blot analysis were from a representative of at least three repeated experiments. CCL18, chemokine (C-C motif) ligand 18; AnxA2, Annexin A2.

expression of E-cadherin was downregulated, whereas levels of vimentin and $\mathrm{N}$-cadherin were upregulated (Fig. 4A, right panels). Immunofluorescence staining of cells indicated the same results (Fig. 4B). Next, the nuclear expression of transcriptional factors was detected to verify the downstream molecules that participate in EMT, which were modulated by AnxA2 in cells. The nuclear expression of Snail was distinctly upregulated in the Scr/MDA-231 cells with $10 \mathrm{ng} / \mathrm{ml}$ rCCL18 stimulation for 3 days when compared with that in the SiAnxA2/MDA-231 cells (Fig. 4C, left panels). The expression of Snail was upregulated in AnxA2/MCF-7 cells with $10 \mathrm{ng} / \mathrm{ml}$ rCCL18 stimulation when compared with the Con/MCF-7 cells (Fig. 4C, right panels). To confirm that AnxA2, as an upstream regulator, plays a role in the Akt/GSK3 $\beta$ signaling pathway during cell chemotaxis, we investigated the influence of AnxA2 expression on CCL18-induced activation of Akt and GSK3 $\beta$. Phosphorylated Akt and GSK3 $\beta$ were assessed after stimulation with $10 \mathrm{ng} / \mathrm{ml}$ rCCL18 for 3 days. The results showed that the phosphorylation of Akt at Ser473 and Thr308 and phosphorylation of GSK3 $\beta$ at Ser 9 were decreased in the SiAnxA2/MDA-231 cells compared with the Scr/MDA-231 cells (Fig. 4D, left panels). The phosphorylated levels of Akt and GSK $3 \beta$ were increased in AnxA2/MCF-7 cells when compared with the Con/MCF-7 cells (Fig. 4D, right panels). We treated the cells with an inhibitor (LY294002) specific to the Akt/GSK3 $\beta$ signaling pathway to detect the activation of AnxA2 with rCCL18 stimulation for 3 days. The results indicated that the phosphorylation of AnxA2 was reduced in the SiAkt/MDA-231 cells and MDA-MB-231 cells with LY294002 compared with the Scr/MDA-231 cells (Fig. 4E). These results suggest that the PI3K/Akt/GSK3 $\beta /$ Snail signaling pathway is pivotal for AnxA2 to promote CCL18-induced EMT in breast cancer cells.

AnxA2 plays a role in breast cancer metastasis with CCL18 stimulation in vivo. A xenograft transplant model in SCID mice was performed to identify the metastatic properties of breast cancer cells. Colonization in lungs was examined through H\&E staining. As showed in Fig. 5A, the results showed that the number of metastatic tumor nodules was increased in the lungs of mice whose Scr/MDA-231 cells were injected with rCCL18 compared with that in the lungs of mice whose SiAnxA2/MDA-231 cells were injected with rCCL18. Quantitative analysis of the number of metastatic tumor nodules indicated that compared with Scr/MDA-231 cells, the SiAnxA2/MDA-231 cells a 5-fold lower number of metastatic tumor nodules in response to $10 \mathrm{ng} / \mathrm{ml} \mathrm{CCL18} \mathrm{(Fig.} \mathrm{5B).} \mathrm{In}$ our present study, we demonstrated that AnxA2 contributes to CCL18-induced metastasis of human breast cancer. Thus, our results revealed that AnxA2 was vital for CCL18-induced metastasis of human breast cancer cells.

\section{Discussion}

Various nonmalignant stromal cells in the tumor microenvironment play a pivotal role in tumor progression and metastasis (31). CCL18, which is derived from TAMs, is correlated with the invasion, migration, metastasis and poor prognosis of patients with breast cancer $(32,33)$. Decades of research have shown that upregulation of Annexin A2 (AnxA2) is involved in many malignant neoplasms, and is associated with the aggressiveness of breast cancer cells (17,34-36). Furthermore, AnxA2 may also be a significant biomarker for breast cancer diagnosis and a potential target for breast cancer therapy $(37,38)$. In the present study, pathological investigation of breast tissue specimens revealed that compared with adjacent non-tumor tissues, AnxA2 was highly regulated in breast cancer tissues, and AnxA2 was upregulated in $68 \%$ of patients with lymph node metastases and in $32 \%$ of those with non-lymph node metastases. AnxA2 was also highly expressed in $65 \%$ in patients with distant metastases. These findings suggested that AnxA2 was highly expressed in most breast cancer patient tissues and plays a vital role in lymph node and distant metastases. In addition, AnxA2 was upregulated in highly invasive human breast cancer cells. This finding indicated that AnxA2 contributes to the invasion of breast cancer cells. In animal experiments, AnxA2 enhanced the metastasis of human breast cancer cells to lungs of SCID mice with rCCL18 stimulation, which provides molecular evidence that AnxA2 is indispensable for CCL18-induced metastasis of human breast cancer cells in vivo. For tumors to metastasize and invade, neoplastic and endothelial cells must migrate into surrounding tissues (39). In addition, migration is highly associated with infiltration and chemotaxis $(40,41)$. In 


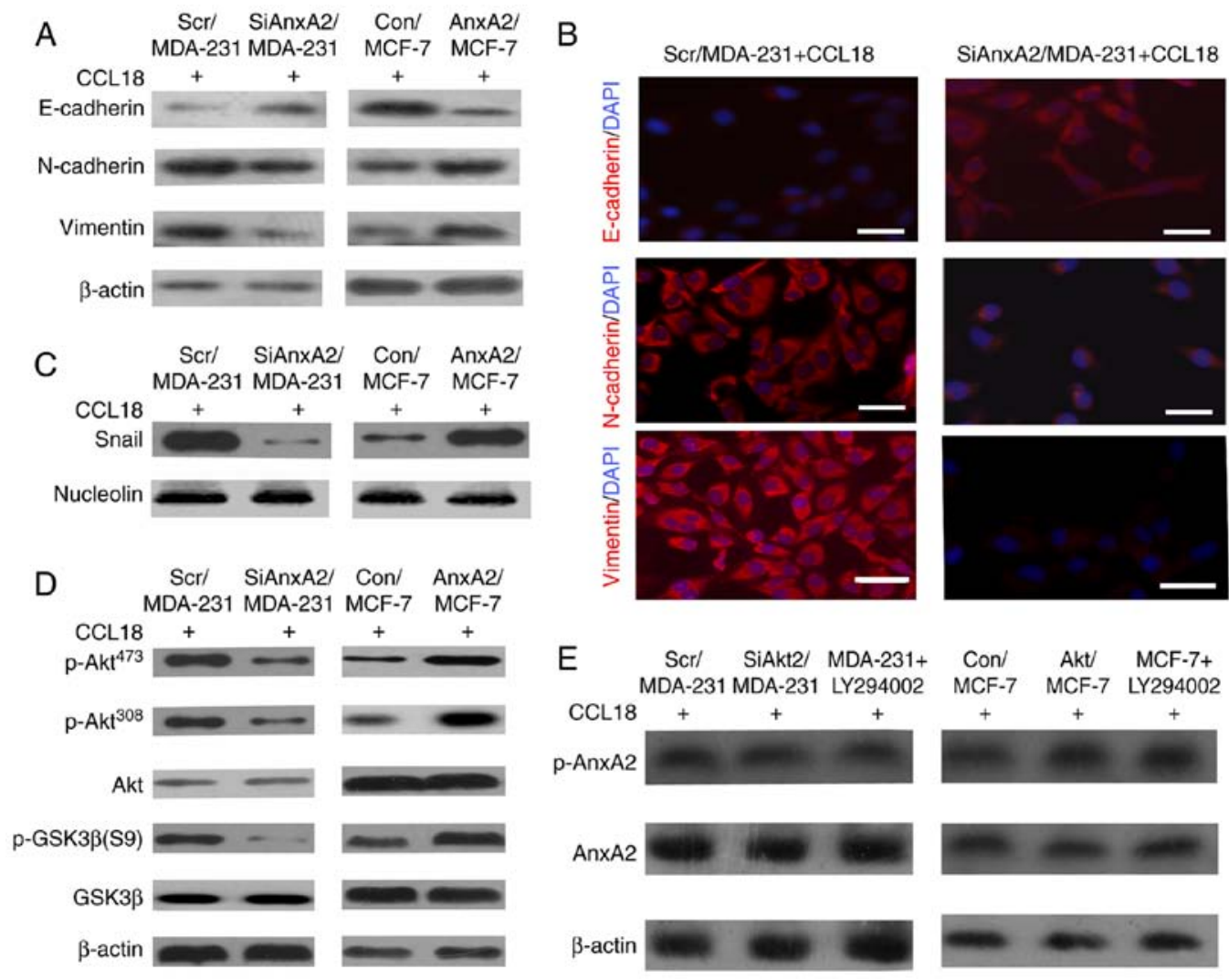

Figure 4. AnxA2 promotes CCL18-induced EMT via the Akt/GSK3 $\beta$ signaling pathway. (A) Expression levels of an epithelial marker (E-cadherin), as well as mesenchymal markers (N-cadherin and vimentin) were assessed by western blot analysis in Scr/MDA-231, SiAnxA2/MDA-231, Con/MCF-7 and AnxA2/MCF-7 cells upon $10 \mathrm{ng} / \mathrm{ml}$ rCCL18 stimulation. $\beta$-actin was used as a loading control. (B) Fluorescence microscopic staining of E-cadherin, $\mathrm{N}$-cadherin and vimentin (red) is indicated in the Scr/MDA-231, SiAnxA2/MDA-231, Con/MCF-7 and AnxA2/MCF-7 cells upon rCCL18 stimulation. Nuclear DNA was stained with DAPI (blue). Scale bar, $20 \mu \mathrm{m}$. (C) Nuclear expression level of Snail in CCL18-induced Scr/MDA231 and SiAnxA2/MDA-231 cells was examined by Western blot analysis. Nucleolin was used as a loading control. rCCL18, $10 \mathrm{ng} / \mathrm{ml}$. (D) Expression levels of p-Akt (Ser473), p-Akt (Thr308), p-GSK3 $\beta$ and GSK3 $\beta$ in the Scr/MDA-231, SiAnxA2/MDA-231, Con/MCF-7 and AnxA2/MCF-7 cells upon rCCL18 stimulation was detected by western blot analysis. Akt or $\beta$-actin was used as a loading control. (E) CCL18-induced MDA-231 and MCF-7 cells were pretreated with LY294002 (20 $\mu$ M) for $1 \mathrm{~h}$. The expression levels of p-AnxA2 and AnxA2 in the Scr/MDA-231, SiAkt/MDA-231, and MDA-231 + LY294002, Con/MCF-7, Akt/MCF-7 and MCF-7 + LY294002 cells were detected by western blot analysis. rCCL18, $10 \mathrm{ng} / \mathrm{ml}$. Data were collected from a representative of at least three independent experiments. CCL18, chemokine (C-C motif) ligand 18; AnxA2, Annexin A2.

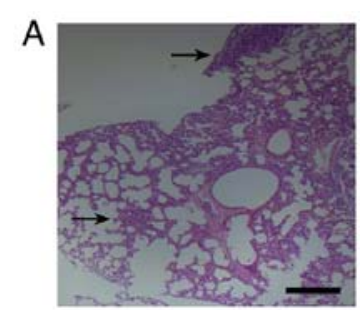

Scr/MDA-231 CCL18

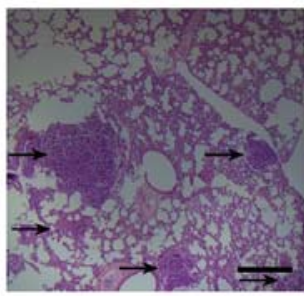

Scr/MDA-231 CCL18 ${ }^{+}$

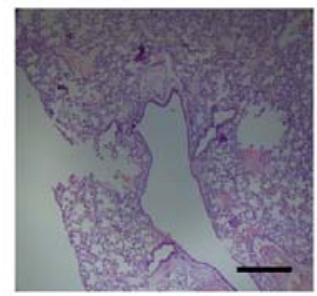

SiAnxA2/MDA-231 CCL18

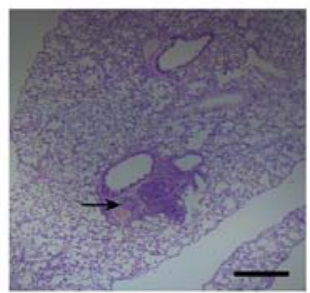

SiAnxA2/MDA-231 CCL18 ${ }^{+}$

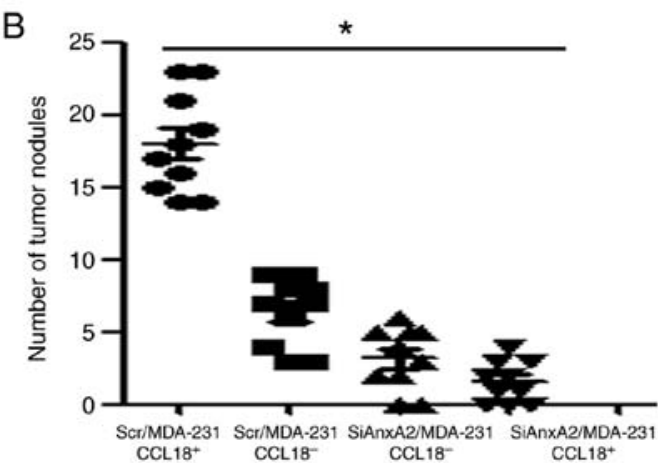

Figure 5. AnxA2 enhances CCL18-induced lung colonization of human breast cancer cells. (A) Micrometastasis in mouse lungs were visualized by hematoxylin and eosin (H\&E) staining. Scale bar, $200 \mu \mathrm{m}$. (B) Lung metastatic nodules were counted and plotted ( $\mathrm{n}=10)$. " $\mathrm{P}<0.05$ (two-way ANOVA). CCL18, chemokine (C-C motif) ligand 18; AnxA2, Annexin A2. 
the present study, upregulation of AnxA2 expression distinctly enhanced the migration ability of the human breast cancer cells upon rCCL18 stimulation in the chemotaxis and scratch assays. These results indicate that AnxA2 enhanced CCL18-induced migration in breast cancer cells. Chemotaxis is the ability of cells to migrate in response to a chemoattractant. Cell migration can be viewed as a multistep of discrete processes that result in net cell-body movement (42). Our study demonstrated that AnxA2 contributed to F-actin polymerization to mediate cytoskeletal rearrangement, providing the driving force for migration and invasion of breast cancer cells. Adhesion contributes to cell movement, and the firm adhesion of tumor cells to the extracellular matrix (ECM) is regulated by integrin $\beta 1$ (26). Our results revealed that silencing of AnxA2 in MDA-MB-231 cells inhibited CCL18-induced phosphorylation of integrin $\beta 1$. Although AnxA2 plays a pivotal role in promoting invasion and migration in breast cancer cells, other chemokines or proteins may also have undiscovered roles. Further research should be performed to determine the undiscovered elements associated with invasion, migration and metastasis in breast cancer cells EMT is indispensable for tumor cell migration, invasion and metastasis in all types of malignant tumors (43). Our previous study (3) showed that MCF-7/Nirl cells increased invasion and migration compared with the MCF-7/Con cells, and the binding of CCL18 to Nirl enhanced EMT in breast cancer cells through the PI3K/Akt/GSK3 $\beta /$ Snail signaling pathway. Moreover, the expression levels of other transcriptional factors (Slug, Twist1, Zeb1 and Zeb2) were stable in the Scr/MDA-231 and SiNir1/MDA-231 cells with or without CCL18 stimulation. Akt contributes to invasion and metastasis, which is a predominant component of the PI3K pathway. Our results showed that AnxA2 depletion in breast cancer cells inhibited CCL18-induced Akt and GSK $3 \beta$ phosphorylation, whereas the expression of Akt and GSK3 $\beta$ was not altered. In addition, AnxA2 enhanced the stabilization of Snail, which is a crucial transcriptional factor of the PI3K/Akt/GSK3 $\beta /$ Snail signaling pathway. In this study, AnxA2 promoted CCL18-induced EMT through the stabilization of Snail via the PI3K/Akt/GSK3//Snail signaling pathway.

In conclusion, our findings indicated that upregulation of AnxA2 promotes breast cancer cell invasion and metastasis. More importantly, we elucidated the molecular mechanism by which AnxA2, as a downstream molecule of CCL18 binding to Nir 1, promotes the progression of breast cancer cell lines. Thus, our research suggests a novel mechanism for CCL18-induced breast cancer cell invasion and metastasis and indicates a valuable prognostic marker and novel therapeutic target for breast cancer.

\section{Acknowledgements}

Not applicable.

\section{Funding}

This study was supported by the National Natural Scientific Foundation of China (nos. 81672631, 81872163, 81072068, 81472365 and 81572578), The Young and Middle-Aged Scientists Research Awards Foundation of Shandong Province (2010BSB14050), the Foundation of Shandong Educational
Committee (J14LK13), the Scientific Foundation of Shandong Province (ZR2014HM003 and ZR2015HM028) and the Shandong Province Outstanding Youth Scientist Foundation Plan (BS2013YY020).

\section{Availability of data and materials}

The datasets used during the present study are available from the corresponding author upon reasonable request.

\section{Authors' contributions}

BZ conceived and designed the study. CZ, SZ, ZY, ZD and RW performed the experiments and collected and analyzed the data. CZ, SZ and BZ wrote the manuscript. All authors read and approved the manuscript and agree to be accountable for all aspects of the research in ensuring that the accuracy or integrity of any part of the work are appropriately investigated and resolved.

\section{Ethics approval and consent to participate}

Patient samples were collected according to a protocol approved by the Institutional Review Board, and patients provided signed consent for use of their tissue specimens in the present study. The study protocol was reviewed and approved by the Weifang Medical University Ethics Committee (approval no. 045, 26-Feb-2016). The study protocol for the animal study was reviewed and also approved by Weifang Medical University Ethics Committee (approval no. 045, 26-Feb-2016).

\section{Patient consent for publication}

Not applicable.

\section{Competing interests}

The authors declare that they have no competing interests.

\section{References}

1. Liu Y, Ji R, Li J, Gu Q, Zhao X, Sun T, Wang J, Li J, Du Q and Sun B: Correlation effect of EGFR and CXCR4 and CCR7 chemokine receptors in predicting breast cancer metastasis and prognosis. J Exp Clin Cancer Res 29: 16, 2010.

2. Wang CZ, Yuan P and Li Y: MiR-126 regulated breast cancer cell invasion by targeting ADAM9. Int J Clin Exp Pathol 8: 6547-6553, 2015.

3. Zhang B, Yin C, Li H, Shi L, Liu N, Sun Y, Lu S, Liu Y, Sun L, Li X, et al: Nirl promotes invasion of breast cancer cells by binding to chemokine (C-C motif) ligand 18 through the $\mathrm{PI} 3 \mathrm{~K} / \mathrm{Akt} / \mathrm{GSK} 3 \beta /$ Snail signalling pathway. Eur J Cancer 49: 3900-3913, 2013

4. Müller A, Homey B, Soto H, Ge N, Catron D, Buchanan ME, McClanahan T, Murphy E, Yuan W, Wagner SN, et al: Involvement of chemokine receptors in breast cancer metastasis. Nature 410: 50-56, 2001.

5. Harvey JR, Mellor P, Eldaly H, Lennard TW, Kirby JA and Ali S: Inhibition of CXCR4-mediated breast cancer metastasis: A potential role for heparinoids? Clin Cancer Res 13: 1562-1570, 2007.

6. Schraufstatter IU, Zhao M, Khaldoyanidi SK and Discipio RG: The chemokine CCL18 causes maturation of cultured monocytes to macrophages in the M2 spectrum. Immunology 135: 287-298, 2012. 
7. Chen P, Li K, Liang Y, Li L and Zhu X: High NUAK1 expression correlates with poor prognosis and involved in NSCLC cells migration and invasion. Exp Lung Res 39: 9-17, 2013.

8. Zhang F, Wang Z, Yuan J, Wei X, Tian R and Niu R: RNAi-mediated silencing of Anxa2 inhibits breast cancer cel proliferation by downregulating cyclin D1 in STAT3-dependent pathway. Breast Cancer Res Treat 153: 263-275, 2015.

9. Wang T, Yuan J, Zhang J, Tian R, Ji W, Zhou Y, Yang Y, Song W, Zhang F and Niu R: Anxa2 binds to STAT3 and promotes epithelial to mesenchymal transition in breast cancer cells Oncotarget 6: 30975-30992, 2015.

10. Kpetemey M, Dasgupta S, Rajendiran S, Das S, Gibbs LD, Shetty P, Gryczynski Z and Vishwanatha JK: MIEN1, a novel interactor of Annexin A2, promotes tumor cell migration by enhancing AnxA2 cell surface expression. Mol Cancer 14: 156, 2015.

11. Zheng L, Foley K, Huang L, Leubner A, Mo G, Olino K, Edil BH, Mizuma M, Sharma R, Le DT, et al: Tyrosine 23 phosphorylation-dependent cell-surface localization of annexin A2 is required for invasion and metastases of pancreatic cancer. PLoS One 6: e19390, 2011.

12. Keklikoglou I, Hosaka K, Bender C, Bott A, Koerner C, Mitra D, Will R, Woerner A, Muenstermann E, Wilhelm H, et al: MicroRNA-206 functions as a pleiotropic modulator of cell proliferation, invasion and lymphangiogenesis in pancreatic adenocarcinoma by targeting ANXA2 and KRAS genes. Oncogene 34: 4867-4878, 2015.

13. Mittal P, Klingler-Hoffmann M, Arentz G, Winderbaum L, Kaur G, Anderson L, Scurry J, Leung Y, Stewart CJ, Carter J, et al: Annexin A2 and alpha actinin 4 expression correlates with metastatic potential of primary endometrial cancer. Biochim Biophys Acta Proteins Proteom 1865: 846-857, 2017.

14. Liu W and Hajjar KA: The annexin A2 system and angiogenesis. Biol Chem 397: 1005-1016, 2016.

15. Ren XL, He GY, Li XM, Men H, Yi LZ, Lu GF, Xin SN, Wu PX, Li YL, Liao WT, et al: MicroRNA-206 functions as a tumor suppressor in colorectal cancer by targeting FMNL2. J Cancer Res Clin Oncol 142: 581-592, 2016.

16. Shi H, Xiao L, Duan W, He H, Ma L, Da M, Duan Y, Wang Q, $\mathrm{Wu} \mathrm{H}$, Song X and Hou Y: ANXA2 enhances the progression of hepatocellular carcinoma via remodeling the cell motility associated structures. Micron 85: 26-33, 2016.

17. Zhang F, Liu Y, Wang Z, Sun X, Yuan J, Wang T, Tian R, Ji W, $\mathrm{Yu}$ M, Zhao Y and Niu R: A novel Anxa2-interacting protein Ebp1 inhibits cancer proliferation and invasion by suppressing Anxa2 protein level. Mol Cell Endocrinol 411: 75-85, 2015

18. Zhang F, Zhang L, Zhang B, Wei X, Yang Y, Qi RZ, Ying G, Zhang $\mathrm{N}$ and Niu R: Anxa2 plays a critical role in enhanced invasiveness of the multidrug resistant human breast cancer cells J Proteome Res 8: 5041-5047, 2009

19. Zhang F, Zhang H, Wang Z, Yu M, Tian R, Ji W, Yang Y and Niu R: P-glycoprotein associates with Anxa2 and promotes invasion in multidrug resistant breast cancer cells. Biochem Pharmacol 87: 292-302, 2014.

20. Zhang HC, Zhang F, Wu B, Han JH, Ji W, Zhou Y and Niu RF: Identification of the Interaction between P-Glycoprotein and Anxa2 in multidrug-resistant human breast cancer cells. Cancer Biol Med 9: 99-104, 2012.

21. Sulaiman A, Yao Z and Wang L: Re-evaluating the role of epithelial-mesenchymal-transition in cancer progression. J Biomed Res 32: 81-90, 2018.

22. Thomson S, Petti F, Sujka-Kwok I, Mercado P, Bean J, Monaghan M, Seymour SL, Argast GM, Epstein DM and Haley JD: A systems view of epithelial-mesenchymal transition signaling states. Clin Exp Metastasis 28: 137-155, 2011.

23. Zhang B, Ma Y, Guo H, Sun B, Niu R, Ying G and Zhang N: Akt2 is required for macrophage chemotaxis. Eur J Immunol 39: 894-901, 2009
24. Sun R, Gao P, Chen L, Ma D, Wang J, Oppenheim JJ and Zhang N: Protein kinase $\mathrm{C}$ zeta is required for epidermal growth factor-induced chemotaxis of human breast cancer cells. Cancer Res 65: 1433-1441, 2005.

25. Yang Y, Wu N, Wang Z, Zhang F, Tian R, Ji W, Ren X and Niu R: Rack1 mediates the interaction of P-glycoprotein with Anxa2 and regulates migration and invasion of multidrug-resistant breast cancer cells. Int J Mol Sci 17: pii: E1718, 2016.

26. Zhang B, Gu F, She C, Guo H, Li W, Niu R, Fu L, Zhang N and Ma Y: Reduction of Akt2 inhibits migration and invasion of glioma cells. Int J Cancer 125: 585-595, 2009.

27. Antón IM, Jones GE, Wandosell F, Geha R and Ramesh N: WASP-interacting protein (WIP): Working in polymerisation and much more. Trends Cell Biol 17: 555-562, 2007.

28. Etienne-Manneville S and Hall A: Rho GTPases in cell biology. Nature 420: 629-635, 2002

29. Ananthakrishnan R and Ehrlicher A: The forces behind cell movement. Int J Biol Sci 3: 303-317, 2007.

30. Doerr ME and Jones JI: The roles of integrins and extracellular matrix proteins in the insulin-like growth factor I-stimulated chemotaxis of human breast cancer cells. J Biol Chem 271: 2443-2447, 1996.

31. Liu Y, Sun R, Wan W, Wang J, Oppenheim JJ, Chen L and Zhang N: The involvement of lipid rafts in epidermal growth factor-induced chemotaxis of breast cancer cells. Mol Membr Biol 24: 91-101, 2007.

32. Chen J, Yao Y, Gong C, Yu F, Su S, Chen J, Liu B, Deng H, Wang F, Lin L, et al: CCL18 from tumor-associated macrophages promotes breast cancer metastasis via PITPNM3. Cancer Cell 19: 541-555, 2011.

33. Lin L, Chen YS, Yao YD, Chen JQ, Chen JN, Huang SY, Zeng YJ, Yao HR, Zeng SH, Fu YS and Song EW: CCL18 from tumor-associated macrophages promotes angiogenesis in breast cancer. Oncotarget 6: 34758-34773, 2015.

34. Wu B, Zhang F, Yu M, Zhao P, Ji W, Zhang H, Han J and Niu R: Up-regulation of Anxa2 gene promotes proliferation and invasion of breast cancer MCF-7 cells. Cell Prolif 45: 189-198, 2012

35. Liu X, Ma D, Jing X, Wang B, Yang W and Qiu W: Overexpression of ANXA2 predicts adverse outcomes of patients with malignant tumors: A systematic review and meta-analysis. Med Oncol 32: 392, 2015 .

36. Sharma MR, Koltowski L, Ownbey RT, Tuszynski GP and Sharma MC: Angiogenesis-associated protein annexin II in breast cancer: Selective expression in invasive breast cancer and contribution to tumor invasion and progression. Exp Mol Pathol 81: 146-156, 2006.

37. Sharma MC and Sharma M: The role of annexin II in angiogenesis and tumor progression: A potential therapeutic target. Curr Pharm Des 13: 3568-3575, 2007.

38. Kesavan K, Nath G and Pandit JK: Sodium alginate based mucoadhesive system for gatifloxacin and its in vitro antibacterial activity. Sci Pharm 78: 941-957, 2010.

39. Hood JD and Cheresh DA: Role of integrins in cell invasion and migration. Nat Rev Cancer 2: 91-100, 2002.

40. Noritake J, Watanabe T, Sato K, Wang S and Kaibuchi K: IQGAP1: A key regulator of adhesion and migration. J Cell Sci 118: 2085-2092, 2005

41. Lu S, Niu N, Guo H, Tang J, Guo W, Liu Z, Shi L, Sun T, Zhou F, $\mathrm{Li} \mathrm{H}$, et al: ARK5 promotes glioma cell invasion, and its elevated expression is correlated with poor clinical outcome. Eur J Cancer 49: 752-763, 2013.

42. Horwitz AR and Parsons JT: Cell migration-movin' on. Science 286: 1102-1103, 1999.

43. Thiery JP, Acloque H, Huang RY and Nieto MA: Epithelialmesenchymal transitions in development and disease. Cell 139: 871-890, 2009. 